\title{
Chest computed tomography imaging improves potential lung donor assessment
}

Jason M. Gauthier, MD, ${ }^{\mathrm{a}}$ Andrew J. Bierhals, MD, MPH, ${ }^{\mathrm{b}}$ Jingxia Liu, MS, PhD, ${ }^{\mathrm{c}}$ Keki R. Balsara, MD, ${ }^{\mathrm{d}}$ Christine Frederiksen, MS, ${ }^{a}$ Emily Gremminger, BA, ${ }^{a}$ Ramsey R. Hachem, MD, ${ }^{e}$ Chad A. Witt, MD, Elbert P. Trulock, MD, ${ }^{\mathrm{e}}$ Derek E. Byers, MD, PhD, ${ }^{\mathrm{e}}$ Roger D. Yusen, MD, MPH, ${ }^{\mathrm{e}}$ Patrick R. Aguilar, MD, Gary Marklin, MD, ${ }^{\mathrm{f}}$ Ruben G. Nava, MD, ${ }^{\mathrm{a}}$ Benjamin D. Kozower, MD, MPH, ${ }^{\mathrm{a}}$ Michael K. Pasque, MD, ${ }^{\mathrm{a}}$ Bryan F. Meyers, MD, MPH, ${ }^{a}$ G. Alexander Patterson, MD, ${ }^{a}$ Daniel Kreisel, MD, PhD, ${ }^{a, g}$ and Varun Puri, MD, MSCI ${ }^{\mathrm{a}}$

\section{ABSTRACT}

Objective: Chest computed tomography (CT) imaging is being increasingly used for potential lung donor assessment. However, the efficacy of CT imaging in this setting remains unknown. We hypothesize that chest CT imaging independently affects the decision-making process in donor lung utilization.

Methods: We conducted a retrospective cohort study of all adult donation after brain death donors managed through our local organ procurement organization from June 2011 to November 2016. An experienced thoracic radiologist independently reviewed donor chest $\mathrm{CT}$ and chest $\mathrm{x}$-ray images in a blinded, standardized manner to determine the presence of structural lung disease (eg, emphysema, interstitial lung disease [ILD]) and acute abnormalities (eg, traumatic lung injury [TLI]). Distinct models of lung utilization were fit to groups with initial partial pressure of oxygen $\left(\mathrm{PaO}_{2}\right) \leq 300 \mathrm{~mm} \mathrm{Hg}$ (suboptimal) and $\mathrm{iPaO}_{2}>300 \mathrm{~mm}$ $\mathrm{Hg}$ (optimal).

Results: The organ procurement organization managed 753 donors during the study period, with a lung utilization rate ([lung donors/all organ donors $] \times 100$ ) of $36.5 \%$ ( 275 of 753 ). Four hundred forty-five $(59.1 \%)$ donors received chest CT imaging, revealing emphysema (13.7\%), ILD (2.5\%), and TLI $(7.2 \%)$. In univariate analysis, findings of TLI (odds ratio [OR], 2.23; 95\% confidence interval [CI], 1.08-4.61) were positively associated with lung utilization, whereas findings of emphysema (OR, 0.18 ; CI, 0.08-0.40) were negatively associated with utilization. In multivariate analysis, CT findings of emphysema (OR, 0.21 ; CI $0.08-0.54$ ) remained negatively associated with utilization. No potential donors with CT findings of ILD became lung donors. After controlling for chest $\mathrm{x}$-ray findings, chest $\mathrm{CT}$ imaging findings of structural lung disease remained negatively associated with utilization $(P=.0001)$. Lung utilization rate in the suboptimal and optimal $\mathrm{iPaO}_{2}$ populations was $35.1 \%$ and $41.4 \%$, respectively, and CT findings of emphysema had a significant association with nonutilization in both groups.

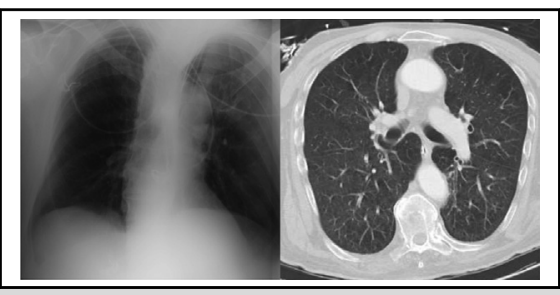

CT findings of structural lung disease are negatively associated with lung utilization.

\section{Central Message}

Chest CT imaging is an important adjunct to conventional lung donor assessment criteria, because findings of structural lung disease (eg, emphysema) have a significant negative association with lung utilization.

\section{Perspective}

Chest CT imaging is being increasingly used for potential lung donor assessment, however, the efficacy of CT in this setting remains unknown. We analyzed the data from 753 potential lung donors to identify determinants of lung utilization.

See Commentaries on pages 1719 and 1720.

\footnotetext{
From the Divisions of a Cardiothoracic Surgery, ${ }^{\mathrm{c}}$ Public Health Sciences, Department of Surgery, and ${ }^{\mathrm{b}}$ Diagnostic Radiology, Mallinckrodt Institute of Radiology; ${ }^{\mathrm{e}}$ Division of Pulmonary and Critical Care Medicine, Department of Medicine, ${ }^{\mathrm{f}}$ Mid-America Transplant, and ${ }^{\mathrm{g}}$ Department of Pathology and Immunology, Washington University School of Medicine, St Louis, Mo; and ${ }^{\mathrm{d}}$ Department of Cardiac Surgery, Vanderbilt University School of Medicine, Nashville, Tenn.

J.M.G. is supported by the U.S. National Institutes of Health (NIH) T32 HL007776, V.P. is supported by NIH K07 CA178120, D.K. is supported by the U.S. Department of Veterans Affairs 1I01BX002730, NIH R01 HL094601, and NIH 1P01AI116501. A.J.B. is supported by NIH R01 HL131961 and NIH R01 DC011311.
}

Read at the 98th Annual Meeting of The American Association for Thoracic Surgery, San Diego, California, April 28-May 1, 2018.

Received for publication April 28, 2018; revisions received Oct 1, 2018; accepted for publication Nov 10, 2018; available ahead of print Feb 13, 2019.

Address for reprints: Varun Puri, MD, MSCI, Division of Cardiothoracic Surgery, Washington University School of Medicine, Campus Box 8234, 660 South Euclid Ave, St Louis, MO 63110 (E-mail: varunpuri@wustl.edu).

0022-5223/\$36.00

Copyright (C) 2018 by The American Association for Thoracic Surgery

https://doi.org/10.1016/j.jtcvs.2018.11.038 


\section{Abbreviations and Acronyms}

$\mathrm{COPD}=$ chronic obstructive pulmonary disease

$\mathrm{CT}=$ computed tomography

$\mathrm{CXR}=$ chest $\mathrm{X}$-ray

ILD = interstitial lung disease

$\mathrm{iPaO} 2=$ initial partial pressure of oxygen

LT = lung transplantation

MTS $=$ Mid-America Transplant

$\mathrm{OPO}=$ organ procurement organization

$\mathrm{PaO} 2=$ partial pressure of oxygen

SDCF $=$ specialized donor care facility

TLI = traumatic lung injury

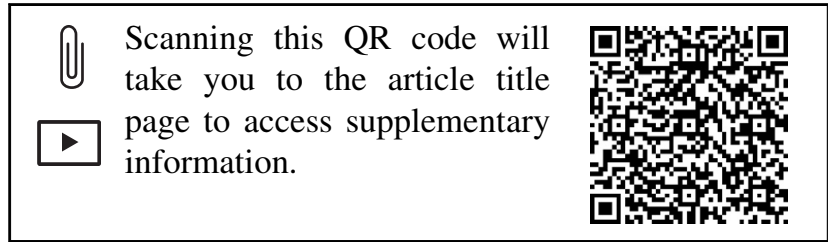

Conclusions: In the evaluation of potential lung donors, chest CT imaging findings of structural lung disease, such as emphysema and ILD, have a significant negative association with lung utilization. Our findings suggest that chest CT imaging might be an important adjunct to conventional lung donor assessment criteria. (J Thorac Cardiovasc Surg 2019;157:1711-8)

Lung transplantation (LT) improves length and quality of life in patients with end stage lung disease. Unfortunately, the patients needing LT far outnumber the number of organs available, as evidenced by a waitlist mortality rate of $15 \%$ to $20 \%{ }^{1}$ In recent years there has been an increase in the number of LTs in the United States; however, the length of the waitlist is largely unchanged and waitlist mortality has increased. ${ }^{2}$ Numerous strategies of lung screening and medical optimization have been proposed with the goal of increasing the donor pool. ${ }^{1,3}$ Because the 5-year survival rate after $\mathrm{LT}$ is only $54 \%$, such efforts to increase the number of lungs used for transplantation must be carefully weighed against the risk of transplanting lungs with poor function. ${ }^{4}$

When evaluating donor lung suitability for transplantation, the only radiographic study traditionally ordered is a chest $\mathrm{x}$-ray (CXR). Conventional donor assessment criteria require the CXR to be free of pathology for lungs to be deemed appropriate for transplantation. "With the gradual acceptance of extended criteria for "marginal donors," lungs without a normal CXR, among other factors, are being increasingly utilized. ${ }^{5-7}$ Although most studies have shown no difference in recipient outcomes between those who had extended criteria compared with traditional criteria donor lungs, others have shown a higher incidence of primary graft dysfunction and mortality among recipients. ${ }^{8,9}$ This discrepancy highlights the need for a thorough and validated donor selection process, particularly with regard to donors who have marginal oxygenation and abnormal imaging. Furthermore, the efficacy of CXR in this setting remains unclear, with some studies reporting a low sensitivity for detecting the presence of chronic lung diseases, such as emphysema. ${ }^{10,11}$

Our local organ procurement organization (OPO), MidAmerica Transplant (MTS), is one of a few specialized donor care facilities (SDCFs) in the country where donation after brain death donors are transported to the facility for medical optimization and organ procurement. MTS has a freestanding computed tomography (CT) scanner and liberally obtains a chest $\mathrm{CT}$ scan for many potential lung donors. Although previous studies have examined chest CT imaging as a means of size-matching donors and recipients, no literature exists on the role of this imaging modality in the preoperative evaluation of lungs donors. ${ }^{12}$ The purpose of this study was to examine the efficacy of chest CT imaging in the evaluation of potential lung donors. We hypothesized that chest CT imaging independently affects the decisionmaking process in donor lung utilization.

\section{METHODS \\ Data Collection and Study Population}

We performed a retrospective analysis of data obtained from MTS's electronic medical records. The study was approved by the Washington University institutional review board (approval date: February 17, 2017; number: 201702077). All donation after brain death donors transferred to MTS between June 1, 2011, and November 31, 2016, were identified. Exclusion criteria included age younger than 18 years and positive hepatitis $\mathrm{B}$ or hepatitis $\mathrm{C}$ serology. Donor demographic and clinical data were retrospectively reviewed and compiled into a secure database. Because a partial pressure of oxygen $\left(\mathrm{PaO}_{2}\right)>300 \mathrm{~mm} \mathrm{Hg}$ on $100 \%$ fraction of inspired oxygen and positive end-expiratory pressure of $5 \mathrm{~cm} \mathrm{H}_{2} \mathrm{O}$ is generally considered adequate gas exchange for donor lungs, we stratified potential donors presenting to MTS with an initial $\mathrm{PaO}_{2}\left(\mathrm{iPaO}_{2}\right) \leq 300 \mathrm{~mm} \mathrm{Hg}$ as "suboptimal," whereas those who presented with $\mathrm{iPaO}_{2}>300 \mathrm{~mm}$ HG were categorized as "optimal." The mean best $\mathrm{PaO}_{2}$ was defined by the highest recorded $\mathrm{PaO}_{2}$ between arrival to MTS and the time of aortic crossclamp. The bronchoscopy finding of "repooling secretions" was defined as reaccumulating secretions in a lobar bronchus after suctioning during a therapeutic bronchoscopy.

For donors who underwent a chest CT scan while at MTS, the CT and CXR images, when available, were independently reviewed by an experienced thoracic radiologist (A.J.B.), who was blinded to donor history and outcome. The radiologist interpreted all chest CT and CXR images separately and remained blinded to all other imaging findings while interpreting a given study. Before initiating the study, a forum of experienced lung transplant providers including surgeons, pulmonary physicians, and thoracic radiologists was assembled to develop an objective protocol for evaluating chest CT images. Images were analyzed for the presence of the following pathology: pneumonia, aspiration, pleural effusion, mucus 
plugging, atelectasis, pneumothorax, interstitial lung disease (ILD), emphysema, and traumatic lung injury (TLI). Each pathological finding was reported as a standardized categorical variable (ie, $0=$ absent, $1=$ present $)$.

\section{Statistical Analysis}

Demographic and clinical data were summarized using descriptive statistics. Lung "utilization" was defined as 1 or more donor lungs being procured for transplantation. Relevant variables were included in a univariate logistic regression model to assess the binary outcome of lung utilization/ nonutilization from potential donors; these variables included donor demographic characteristics and medical history, relevant in-patient data, and chest CT imaging findings. Multivariate regression models through stepwise selection were fitted to assess independent determinants of lung utilization. A significance level of . 3 was required to allow a variable into the model, and a significance level of .1 was required for a variable to stay in the model. We tested model assumptions and performed regression diagnostics. We did not conduct a previous sample size requirement estimates/power calculation because we had a convenience sample and assumed that hundreds of patients would provide adequate power for our analysis. The Cochran-Mantel-Haenszel test was applied to determine the association between $\mathrm{CT}$ variables and lung utilization after controlling for CXR findings. The $\chi^{2}$ test was applied to select CT findings and lung utilization. All tests were conducted in a 2-sided manner and the significance level was set at .05. All analyses were conducted using SAS version 9.2 (SAS Institute Inc, Cary, NC).

\section{RESULTS}

\section{Potential Donor Population}

Seven hundred fifty-three potential lung donors met inclusion criteria during the study period. Of these, lungs were accepted for transplantation from 275 donors, yielding a lung utilization rate of $36.5 \%$. Twelve of the $275(4.36 \%)$ donors were single-lung donors and the remainder were double-lung donors. On arrival to MTS (SDCF), the mean $\mathrm{iPaO}_{2} \pm$ standard deviation on $100 \%$ fraction of inspired oxygen and positive end-expiratory pressure of $5 \mathrm{~cm} \mathrm{H}_{2} \mathrm{O}$ was $204 \pm 141 \mathrm{~mm} \mathrm{Hg}$. In total, 584 of the 753 (77.6\%) potential donors presented with suboptimal $\mathrm{PaO}_{2}(\leq 300 \mathrm{~mm}$ $\mathrm{Hg})$ and $169(22.4 \%)$ presented with optimal $\mathrm{iPaO}_{2}$ ( $>300 \mathrm{~mm} \mathrm{Hg}$ ). The mean best $\mathrm{PaO}_{2}$ was $406 \pm 151 \mathrm{~mm} \mathrm{Hg}$.

Four hundred forty-five $(59.1 \%)$ potential donors received a chest CT scan while at MTS. The most common finding on chest CT imaging was atelectasis (202 of 445; $45.4 \%$ ), followed by pleural effusion (179 of 445; $40.2 \%$ ). Structural lung disease was found in 69 potential donors (69 of $445115.5 \%$ ), including emphysema (61 of $445 ; 13.7 \%)$ and/or ILD (11 of $445 ; 2.5 \%)$. Potential donor baseline characteristics, in-patient data, and chest CT imaging findings are summarized in Table 1.

\section{Determinants of Lung Utilization}

Univariate logistic regression analysis was conducted to detect associations between lung utilization and the covariates of interest, as listed in Table 1. In univariate analysis advanced age, elevated body mass index, and smoking history ( $\geq 20$ pack-years) were all negatively associated with
TABLE 1. Baseline characteristics and chest CT imaging findings of all potential lung donors

\begin{tabular}{|c|c|}
\hline Characteristic & Value \\
\hline \multicolumn{2}{|l|}{ Demographic } \\
\hline Age, y & $43.0 \pm 15.6$ \\
\hline Male sex & $419(55.6)$ \\
\hline BMI & $28.6 \pm 7.8$ \\
\hline Asthma* & $91(12.1)$ \\
\hline COPD* & $34(4.5)$ \\
\hline Smoking history*, $\dagger$ & $200(26.7)$ \\
\hline CXR & 749 (99.5) \\
\hline Chest CT imaging & $565(75.0)$ \\
\hline \multicolumn{2}{|l|}{ In-patient history } \\
\hline Current antibiotic therapy & $527(70.0)$ \\
\hline Sputum grain stain positive* & $347(49.1)$ \\
\hline Sputum culture positive* & $388(55.3)$ \\
\hline Bronchoscopy & $594(78.9)$ \\
\hline Purulent secretions* & $7(1.2)$ \\
\hline Repooling secretions* & $110(18.7)$ \\
\hline Best $\mathrm{PaO}_{2}, \mathrm{~mm} \mathrm{Hg}$ & $405.9 \pm 150.9$ \\
\hline Initial $\mathrm{PaO}_{2} \leq 300 \mathrm{~mm} \mathrm{Hg}$ & $584(77.9)$ \\
\hline Initial $\mathrm{PaO}_{2}>300 \mathrm{~mm} \mathrm{Hg}$ & $169(22.4)$ \\
\hline \multicolumn{2}{|l|}{ Chest CT imaging findings* } \\
\hline Emphysema & $61(13.7)$ \\
\hline Interstitial lung disease & $11(2.5)$ \\
\hline Traumatic lung injury & $32(7.2)$ \\
\hline Pneumothorax & $50(11.2)$ \\
\hline Pneumonia & $144(32.4)$ \\
\hline Aspiration & 87 (19.6) \\
\hline Pleural effusion & $179(40.2)$ \\
\hline Mucus plugging & $113(25.4)$ \\
\hline Atelectasis & $202(45.4)$ \\
\hline
\end{tabular}

Values are presented as mean \pm standard deviation or $\mathrm{n}(\%)$. Baseline characteristics and CT findings of all potential donors managed at MTS from June 2011 to November $2016(\mathrm{n}=753)$. In-patient history refers to time spent at MTS and the transferring facility. Not all patients received bronchoscopy $(\mathrm{n}=594)$ or a chest CT scan ( $\mathrm{n}=445$ ) while at the MTS facility. BMI, Body mass index; COPD, chronic obstructive pulmonary disease; $\mathrm{CXR}$, chest x-ray; $\mathrm{CT}$, computed tomography; $\mathrm{PaO}_{2}$, partial pressure of oxygen. $*$ The denominator for percentages is the sum of patients without missing values. †Includes potential donors with $\geq 20$ pack-year smoking history.

lung utilization. Conversely, higher best $\mathrm{PaO}_{2}$, absence of repooling secretions on bronchoscopy, and current antibiotic therapy were positively associated with likelihood of lung utilization. These findings are summarized in Table 2. Of note, $7.1 \%$ (34 of 476) of nondonors, but none of the lung donors, had a history of chronic obstructive pulmonary disease (COPD). CT findings of emphysema were negatively associated with lung utilization, whereas detection of TLI was positively associated with lung utilization (Table 2). No actual lung donors had a history of COPD or CT findings of ILD, thus the model was not convergent and measures of statistical significance could not be generated for these covariates.

A stepwise multivariate regression analysis was done for the chest $\mathrm{CT}$ imaging findings described. We noted that younger age and higher best $\mathrm{PaO}_{2}$ were highly associated 
TABLE 2. Determinants of lung donation on the basis of univariate logistic regression and stepwise multivariate regression analysis

\begin{tabular}{|c|c|c|c|}
\hline \multirow[b]{2}{*}{ Determinant } & \multirow{2}{*}{$\begin{array}{c}\text { Univariate } \\
P \text { value }\end{array}$} & \multicolumn{2}{|c|}{ Multivariate } \\
\hline & & $P$ value & $\begin{array}{c}\text { Odds Ratio } \\
(95 \% \text { CI) }\end{array}$ \\
\hline \multicolumn{4}{|l|}{ Demographic } \\
\hline Age, y & $<.0001$ & $<.0001$ & $0.97(0.95-0.98)$ \\
\hline Female sex & .88 & & \\
\hline BMI & $<.0001$ & & \\
\hline Asthma & .14 & & \\
\hline Smoking history* & $<.0001$ & & \\
\hline CXR & .98 & & \\
\hline Chest CT imaging & .95 & & \\
\hline \multicolumn{4}{|l|}{ In-patient history } \\
\hline Current antibiotic therapy & .01 & & \\
\hline Sputum grain stain positive & .08 & & \\
\hline Sputum culture positive & .37 & & \\
\hline Bronchoscopy & $<.0001$ & & \\
\hline $\begin{array}{l}\text { Absence of purulent } \\
\text { secretions }\end{array}$ & .89 & & \\
\hline $\begin{array}{l}\text { Absence of repooling } \\
\text { secretions }\end{array}$ & .0007 & & \\
\hline $\begin{array}{l}\text { Best } \mathrm{PaO}_{2} \\
\text { Initial } \mathrm{PaO}_{2}\end{array}$ & $\begin{array}{c}<.0001 \\
.13\end{array}$ & $<.0001$ & $1.01(1.01-1.02)$ \\
\hline \multicolumn{4}{|l|}{ Chest $\mathrm{CT}$ imaging findings } \\
\hline Emphysema & $<.0001$ & .0013 & $0.21(0.08-0.54)$ \\
\hline Traumatic lung injury & .03 & & \\
\hline Pneumothorax & .75 & & \\
\hline Pneumonia & .78 & & \\
\hline Aspiration & .47 & & \\
\hline Pleural effusion & .43 & & \\
\hline Mucus plugging & .27 & & \\
\hline Atelectasis & .74 & & \\
\hline
\end{tabular}

Analysis includes all potential donor variables listed in Table 1. A significance level of .3 was required to allow a variable into the model and a significance level of .1 is required to stay in the model. Bold font denotes $P$ values less than .05 . $C I$, Confidence interval; $B M I$, body mass index; $C X R$, chest x-ray; $C T$, computed tomography; $\mathrm{PaO}_{2}$, partial pressure of oxygen. *Includes potential donors with $\geq 20$ pack-year smoking history.

with lung utilization, whereas findings of emphysema on CT scan significantly lowered the likelihood of lung utilization. The results of the multivariate model are also summarized in Table 2. Of note, ILD was not included in the multivariate analysis because no actual lung donors had findings of ILD on CT images. When CT findings of structural lung disease (emphysema and/or ILD) were analyzed using the $\chi^{2}$ test, structural lung disease was negatively associated with lung utilization $(P<.0001)$.

\section{The Role of Chest CT Imaging in Donor Lung Assessment}

Numerous case studies within the database revealed the influence of chest CT imaging on the decision to accept donor lungs. Figure 1 and Figure 2 show the CXR and chest CT imaging of patients with emphysema and ILD, respectively. In each case the potential donor is younger than 65 years of age, achieved an optimal best $\mathrm{PaO}_{2}$ (>300 $\mathrm{mm} \mathrm{Hg}$ ), and CXR shows nonspecific findings, but chest $\mathrm{CT}$ imaging reveals structural lung disease not evident on CXR. Lungs from potential donors shown in Figures 1 and 2 were declined. Conversely, in many instances lungs were utilized despite a chest CT scan revealing obvious lung pathology that was acute, rather than chronic. The lungs in Figure 3, for example, are also from a patient younger than 65 years of age, who achieved an optimal best $\mathrm{PaO}_{2}(>300 \mathrm{~mm} \mathrm{Hg})$, and CXR shows nonspecific pathology, whereas chest CT imaging reveals bilateral pulmonary contusions suggestive of TLI. In this case, the lungs were accepted for transplantation.

Because structural lung disease (emphysema and/or ILD) and TLI can sometimes be seen on CXR, we tested the association between lung utilization and these CT findings while controlling for CXRs that revealed the same pathology. In total 177 of 445 potential donors $(39.8 \%)$ with CT scans had CXR images available for interpretation. CXR revealed structural lung disease in $4.5 \%$ (8 of 177) and TLI in $17.5 \%$ (31 of 177) of potential donors. Among this cohort, CT revealed a higher prevalence of structural lung disease $(12.4 \% ; 22$ of 177) compared with CXR, but a lower prevalence of TLI $(9.0 \% ; 16$ of 177). After controlling for CXR findings of structural lung disease, CT findings of structural lung disease were negatively associated with utilization $(P=.0001$, Cochran-Mantel-Haenszel test). Conversely, after controlling for CXR findings of TLI, CT findings of TLI showed a trend toward a positive association with utilization, although this was not statistically significant $(P=.09)$. The lung utilization rate for subgroups of potential donors on the basis of radiographic findings of structural lung disease and TLI are provided in Table 3 and Table E1, respectively.

\section{Subgroup Analysis on the Basis of $\mathrm{iPaO}_{2}$}

Of the 753 potential donors, $584(77.6 \%)$ had $\mathrm{iPaO}_{2}$ $\leq 300 \mathrm{~mm} \mathrm{Hg}$. Of these 584 potential donors, lungs were accepted from 205 (35.1\%), whereas 70 of $169(41.4 \%)$ of those with $\mathrm{iPaO}_{2}>300$ became lung donors. Univariate and multivariate analyses for the association of covariates with lung utilization in these 2 subgroups largely confirmed the findings of the overall model and are shown in Tables E2 and E3. Chest CT imaging findings of emphysema were associated with nonutilization in both groups.

Multivariate analysis of potential donors with optimal $\mathrm{iPaO}_{2}$ revealed that older age and findings of emphysema were independent negative determinants of lung utilization. In the subgroup with suboptimal $\mathrm{iPaO}_{2}$, higher best $\mathrm{PaO}_{2}$ was an independent positive determinant of lung utilization, whereas a positive smoking history was an independent negative determinant of lung utilization. 


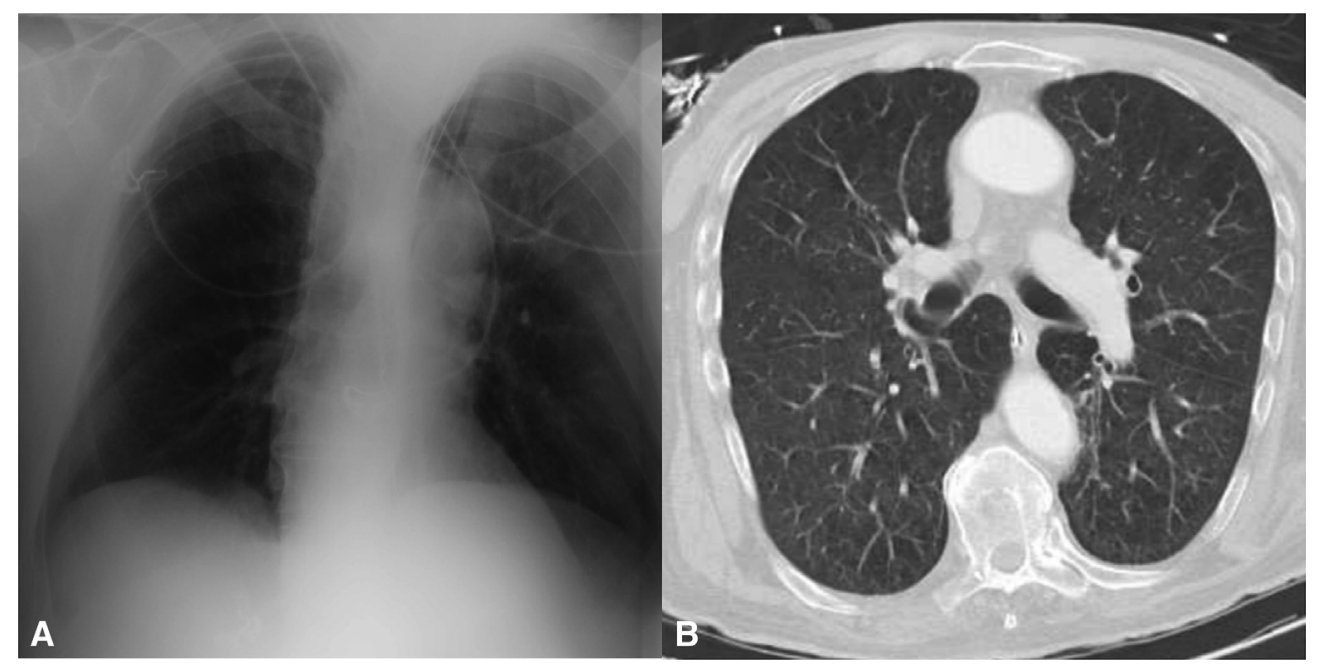

FIGURE 1. Potential lung donor with findings of emphysema on chest computed tomography imaging. The images shown are from a potential donor 64 years of age who died from a cerebrovascular accident. The potential donor achieved a best partial pressure of oxygen of $572 \mathrm{~mm} \mathrm{Hg}$ (optimal) and had a positive smoking history. (A) Chests x-ray image from the potential lung donor is normal. (B) Chest computed tomography scan reveals bilateral lung destruction and hyperexpansion typical of emphysema. The donor lungs were declined for transplantation.

\section{DISCUSSION}

We examined the characteristics of potential lung donors that affect the decision to accept lungs for transplantation and noted that older donor age, lower best $\mathrm{PaO}_{2}$, and findings of emphysema on chest CT imaging were highly associated with declining donor lungs for transplantation. Furthermore, no actual lung donors in the study had a history of COPD or CT findings of ILD. After controlling for CXR findings, CT findings of structural lung disease (emphysema and/or ILD) remained negatively associated with lung utilization. Collectively, our findings suggest that chest CT imaging might be a useful adjunct in the evaluation of potential lung donors, potentially by revealing occult structural lung disease that is missed using traditional criteria (Video 1).

The current criteria for lung donor assessment rely on donor demographic characteristics, medical history, objective measures of pulmonary function, and CXR examination. A clear CXR image and no history of primary pulmonary disease are both required for categorization as an "ideal" lung donor. ${ }^{13}$ Chest CT imaging is not currently part of any lung donor assessment guidelines and no data are available on its efficacy in this setting. At our local OPO the CT scanner is owned by and located

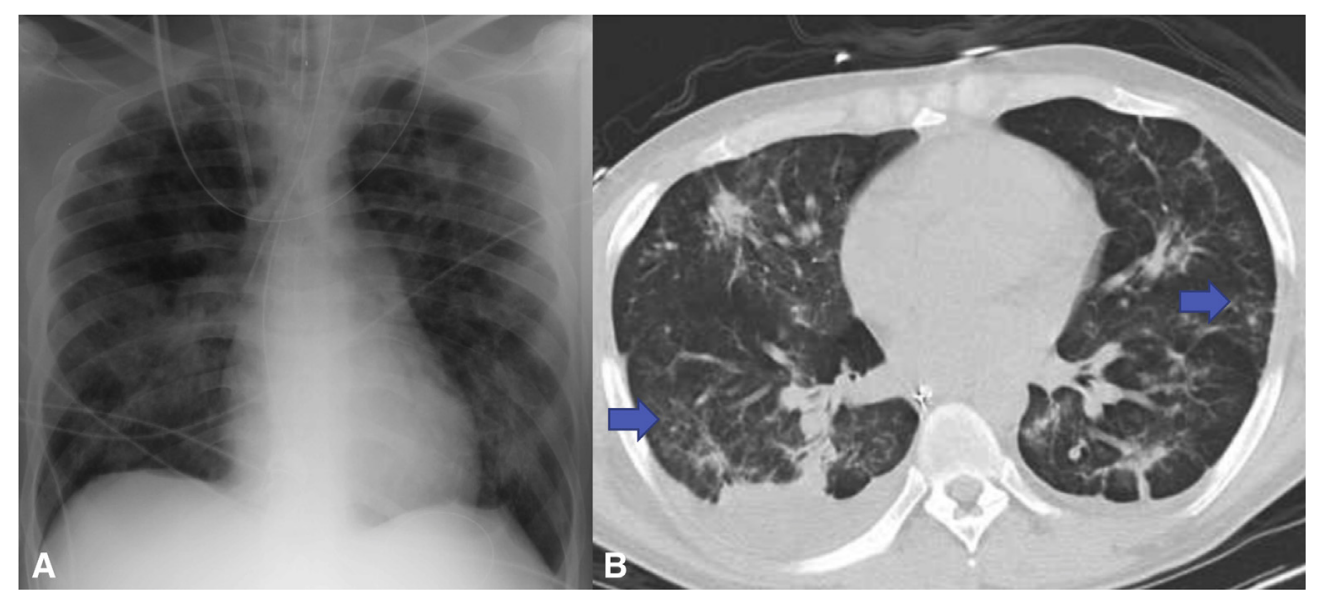

FIGURE 2. Potential lung donor with findings of interstitial lung disease on chest computed tomography imaging. The images shown are from a potential donor 33 years of age who died from drug intoxication. The potential donor achieved a best partial pressure of oxygen of 313 mm Hg (optimal) and had a negative smoking history. (A) Chest X-ray from the potential lung donor reveals mild bilateral patchy opacities without consolidation. (B) Chest computed tomography scan reveals subpleural reticulation and ground glass (arrows) consistent with interstitial lung disease (nonspecific interstitial pneumonia). The donor lungs were declined for transplantation. 


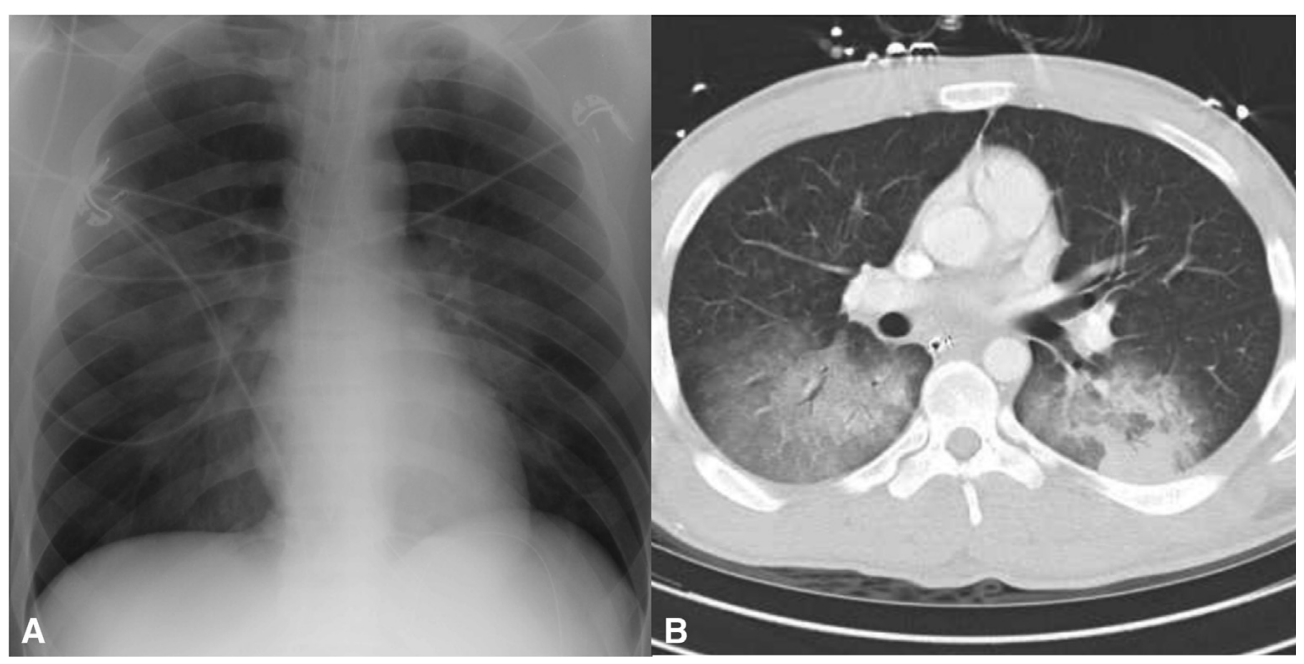

FIGURE 3. Potential lung donor with findings of traumatic lung injury on chest computed tomography imaging. The images shown are from a potential donor 23 years of age who died from head trauma. The potential donor achieved a best partial pressure of oxygen of $500 \mathrm{~mm} \mathrm{Hg}$ (optimal) and had a negative smoking history. (A) Chest $\mathrm{x}$-ray from the potential lung donor reveals bilateral central opacities. (B) Chest computed tomography scan reveals findings consistent with pulmonary contusions and traumatic lung injury. The donor lungs were used for transplantation.

in the SDCF, such that the scans can be obtained quickly and at low cost. As a result, a significant proportion of the potential donors managed by our OPO receive chest CT imaging, which are reviewed by a radiologist and the accepting surgeons. In our experience, donors managed outside of our local OPO's region seldom undergo chest CT imaging, and requests for this imaging by the accepting surgeon on the basis of clinical indications, such as a history of smoking or recent trauma, are often met with resistance. On the surface, this is understandable because of the inconvenience of transporting a donor to the radiology suite, the logistics of juggling requests from multiple accepting centers in a multiorgan donor, and the additional cost involved. The potential benefits of a donor CT scan, however, might outweigh the drawbacks associated with obtaining it.

Chest CT imaging has been shown to be superior to CXR imaging in multiple settings, such as detecting $\mathrm{TLI}^{14}$ or pneumonia, ${ }^{15}$ as well as operative planning before single-

TABLE 3. Lung utilization rate on the basis of CT and CXR findings of SLD

\begin{tabular}{lccc}
\hline & \multicolumn{2}{c}{ Lung utilization rate (lung donors/all donors) } & \\
\cline { 2 - 3 } & CXR without SLD & CXR with SLD & Total \\
\hline CT without SLD & $0.55(82 / 150)$ & $0.40(2 / 5)$ & 155 \\
CT with SLD & $0.11(2 / 19)$ & $0.00(0 / 3)$ & 22 \\
Total & 169 & 8 & \\
\hline
\end{tabular}

Cochran-Mantel-Haenszel test reveals a statistically significant association between lung utilization and CT findings of SLD after controlling for CXR findings of SLD $(P=.0001) . C X R$, Chest x-ray; $S L D$, structural lung disease; $C T$, computed tomography.
LT. $^{16}$ The results of the National Lung Cancer Screening Trial revealed that chest $\mathrm{CT}$ imaging also detects lung cancer at a higher incidence than screening CXRs, which translated into reduced lung cancer mortality in this trial. ${ }^{17}$ In our study $26.7 \%$ of potential donors ( 200 of 753 ) had a $>20$ pack-year smoking history, of which $37 \%$ were 55 years of age or older. Because of the increasing utilization of lungs from donors with a significant smoking history, careful screening of donor lungs for lesions indicative of lung cancer further justifies the use of chest CT imaging in this population. ${ }^{18}$

One additional benefit of chest CT imaging in the evaluation of potential lung donors is the ability to measure the chest cavity and lung volumes to accurately size-match a donor to a recipient. Precise donor-recipient size matching is being increasingly recognized as an important component of the selection process, because size mismatch has been

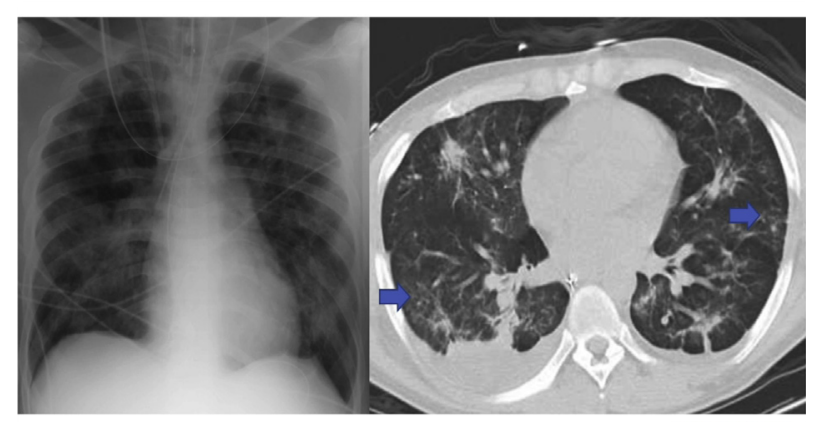

VIDEO 1. The lead author discusses the present study's background, results, and implications for lung donor assessment. Video available at: https://www.jtcvs.org/article/S0022-5223(18)33147-7/fulltext. 
linked to recipient primary graft dysfunction, ${ }^{19}$ bronchiolitis obliterans, ${ }^{20}$ and overall survival. ${ }^{21}$ Traditionally this assessment has relied on donor and recipient height and/ or total lung capacity; using a donor chest CT scan, however, allows for more accurate determination of intrathoracic dimensions. Additionally, CT imaging is frequently used to study liver volume for appropriate donor-recipient matching and additional use of a chest CT scan while an abdominal $\mathrm{CT}$ is being performed might make the effort less onerous and more efficient. Thus, chest CT imaging might be a method of detecting poor-quality or inappropriately sized lungs remotely, before sending a retrieval team to evaluate the organ. There is a substantial cost and risk to personnel associated with flying a retrieval team to evaluate organs, ${ }^{22}$ and improved methods of evaluating potential donors remotely are greatly needed.

Our results remain limited by several factors and should be interpreted with these limitations in mind. Inherent to the design of a retrospective study is the possibility of information bias, limited sample size, and inability to control exposure to a given intervention, such as chest $\mathrm{CT}$ imaging. As evidence of this, only 445 of the 753 $(59.1 \%)$ potential donors in the study period received chest CT imaging at MTS, and we were unable to access images of the 120 donors who received chest CT imaging before being transferred to MTS. Our ability to control for CXR findings was limited by data availability as well, because only 177 of the 445 donors $(39.8 \%)$ with CT scans at MTS also had CXRs available for review. Furthermore, the single radiologist who interpreted all chest CT images and CXRs was blinded to donor history, lung outcome, and other imaging findings; although this is indeed a strength of the study, it might also be interpreted as a weakness because most radiologists interpret images in the setting of patient/donor history. Attempts at associating donor CT findings with recipient outcomes were unfortunately limited by access to CT images, because only 132 of the $221(59.7 \%)$ transplantations done at our institution had CT scans available for interpretation. It should be noted that having an SDCF such as MTS is not typical and might have altered the decision-making in some capacity. Furthermore, our hospital is a high-volume lung transplantation center that uses institutional standards to guide lung donor assessment, and these results might not be generalizable to some centers.

Our study represents the first, to our knowledge, to examine the effect of chest $\mathrm{CT}$ imaging on the lung donor selection process. With CT scanners becoming more prevalent in the United States and the resolution increasingly better, ${ }^{23}$ donor chest CT imaging could realistically become a part of the traditional lung donor selection criteria. Further investigation in the form of prospective studies is needed to determine if improved lung donor selection via chest $\mathrm{CT}$ imaging translates into improved recipient outcomes.

\section{CONCLUSIONS}

In the evaluation of potential lung donors, chest CT imaging findings of structural lung disease, such as emphysema and ILD, have a significant negative association with lung utilization. Our findings suggest that chest $\mathrm{CT}$ imaging might be an important adjunct to conventional lung donor assessment criteria.

\section{Conflict of Interest Statement}

D.K. is on the scientific advisory board of Compass Therapeutics. All other authors have nothing to disclose with regard to commercial support.

\section{References}

1. Chang SH, Kreisel D, Marklin GF, Cook L, Hachem RR, Kozower BD, et al. Lung focused resuscitation at a specialized donor care facility improves lung procurement rates. Ann Thorac Surg. 2018;105:1531-6.

2. Valapour M, Skeans MA, Smith JM, Edwards LB, Cherikh WS, Uccellini K, et al. OPTN/SRTR 2015 annual data report: lung. Am J Transplant. 2017; 17(suppl 1):357-424

3. Sommer W, Kühn C, Tudorache I, Avsar M, Gottlieb J, Boethig D, et al. Extended criteria donor lungs and clinical outcome: results of an alternative allocation algorithm. J Heart Lung Transplant. 2013;32:1065-72.

4. Yusen RD, Edwards LB, Dipchand AI, Goldfarb SB, Kucheryavaya AY, Levvey BJ, et al. The registry of the International Society for Heart and Lung Transplantation: thirty-third adult lung and heart-lung transplant report-2016; focus theme: primary diagnostic indications for transplant. J Heart Lung Transplant. 2016;35:1170-84.

5. Sundaresan S, Semenkovich J, Ochoa L, Richardson G, Trulock EP, Cooper JD, et al. Successful outcome of lung transplantation is not compromised by the use of marginal donor lungs. J Thorac Cardiovasc Surg. 1995;109:1075-9; discussion: $1079-80$

6. Aigner C, Winkler G, Jaksch P, Seebacher G, Lang G, Sharokh T, et al. Extended donor criteria for lung transplantation-a clinical reality. Eur J Cardiothorac Surg. 2005;27:757-61

7. Lardinois D, Banysch M, Korom S, Hillinger S, Rousson V, Boehler A, et al. Extended donor lungs: eleven years experience in a consecutive series. Eur $J$ Cardiothorac Surg. 2005;27:762-7.

8. Mulligan MJ, Sanchez PG, Evans CF, Wang Y, Kon ZN, Rajagopal K, et al. The use of extended criteria donors decreases one-year survival in high-risk lung recipients: a review of the united network of organ sharing database. J Thorac Cardiovasc Surg. 2016;152:891-8.e892

9. Pierre AF, Sekine Y, Hutcheon MA, Waddell TK, Keshavjee SH. Marginal donor lungs: a reassessment. J Thorac Cardiovasc Surg. 2002;123:421-7; discussion: 427-8.

10. Thurlbeck WM, Simon G. Radiographic appearance of the chest in emphysema AJR Am J Roentgenol. 1978;130:429-40.

11. Miniati M, Monti S, Stolk J, Mirarchi G, Falaschi F, Rabinovich R, et al. Value of chest radiography in phenotyping chronic obstructive pulmonary disease. Eur Respir J. 2008;31:509-15.

12. Konheim JA, Kon ZN, Pasrija C, Luo Q, Sanchez PG, Garcia JP, et al. Predictive equations for lung volumes from computed tomography for size matching in pulmonary transplantation. J Thorac Cardiovasc Surg. 2016; 151:1163-9.e1.

13. Van Raemdonck D, Neyrinck A, Verleden GM, Dupont L, Coosemans W Decaluwe $\mathrm{H}$, et al. Lung donor selection and management. Proc Am Thorac Soc. 2009;6:28-38.

14. Trupka A, Waydhas C, Hallfeldt KK, Nast-Kolb D, Pfeifer KJ, Schweiberer L. Value of thoracic computed tomography in the first assessment of severely injured patients with blunt chest trauma: results of a prospective study. J Trauma 1997;43:405-11; discussion: 411-2. 
15. Self WH, Courtney DM, McNaughton CD, Wunderink RG, Kline JA. High discordance of chest x-ray and computed tomography for detection of pulmonary opacities in ED patients: implications for diagnosing pneumonia. Am J Emerg Med. 2013;31:401-5.

16. Kazerooni EA, Chow LC, Whyte RI, Martinez FJ, Lynch JP. Preoperative examination of lung transplant candidates: value of chest CT compared with chest radiography. AJR Am J Roentgenol. 1995;165:1343-8.

17. National Lung Screening Trial Research Team, Aberle DR, Adams AM, Berg CD, Black WC, Clapp JD, Fagerstrom RM, et al. Reduced lung-cancer mortality with low-dose computed tomographic screening. $N$ Engl J Med. 2011;365: 395-409.

18. Bonser RS, Taylor R, Collett D, Thomas HL, Dark JH, Neuberger J, et al. Effect of donor smoking on survival after lung transplantation: a cohort study of a prospective registry. Lancet. 2012;380:747-55.

19. Eberlein M, Reed RM, Bolukbas S, Diamond JM, Wille KM, Orens JB, et al. Lung size mismatch and primary graft dysfunction after bilateral lung transplantation. J Heart Lung Transplant. 2015;34:233-40.
20. Eberlein M, Permutt S, Chahla MF, Bolukbas S, Nathan SD, Shlobin OA, et al. Lung size mismatch in bilateral lung transplantation is associated with allograft function and bronchiolitis obliterans syndrome. Chest. 2012; 141:451-60.

21. Eberlein M, Reed RM, Maidaa M, Bolukbas S, Amaoutakis GJ, Orens JB, et al. Donor-recipient size matching and survival after lung transplantation. A cohort study. Ann Am Thorac Soc. 2013;10:418-25.

22. Lindemann J, Dageforde LA, Brockmeier D, Vachharajani N, Scherer M, Chapman W, et al. Organ procurement center allows for daytime liver transplantation with less resource utilization: may address burnout, pipeline and safety for field of transplantation. Am J Transplant. September 24, 2018 [Epub ahead of print].

23. Stockburger WT. CT imaging, then and now: a 30-year review of the economics of computed tomography. Radiol Manage. 2004;26:20-2, 24-7.

Key Words: lung transplantation, organ allocation, surgery, lung diseases 
TABLE E1. Lung utilization rate on the basis of CT and CXR findings of TLI

\begin{tabular}{|c|c|c|c|}
\hline & \multicolumn{2}{|c|}{ Lung utilization rate (lung donors/all donors) } & \multirow[b]{2}{*}{ Total } \\
\hline & CXR without TLI & CXR with TLI & \\
\hline CT without TLI & $0.54(73 / 135)$ & $0.12(3 / 26)$ & 161 \\
\hline CT with TLI & $0.64(7 / 11)$ & $0.60(3 / 5)$ & 16 \\
\hline Total & 146 & 31 & \\
\hline
\end{tabular}

TABLE E2. Univariate and multivariate logistic regression analyses of likelihood of lung donation in potential donors with optimal $\mathrm{iPaO}_{2}$

\begin{tabular}{|c|c|c|c|}
\hline \multirow[b]{2}{*}{ Variable } & \multirow{2}{*}{$\frac{\text { Univariate }}{P \text { value }}$} & \multicolumn{2}{|c|}{ Multivariate } \\
\hline & & $P$ value & $\overline{\text { OR }(95 \% \mathrm{CI})}$ \\
\hline \multicolumn{4}{|l|}{ Demographic } \\
\hline Age, y & $<.0001$ & .008 & $0.96(0.93-0.99)$ \\
\hline Female sex & .33 & & \\
\hline BMI & .23 & & \\
\hline Asthma & .44 & & \\
\hline Smoking history* & .0024 & & \\
\hline Chest CT imaging & .35 & & \\
\hline \multicolumn{4}{|l|}{ In-patient history } \\
\hline Current antibiotic therapy & .75 & & \\
\hline Sputum grain stain positive & .13 & & \\
\hline Sputum culture positive & .55 & & \\
\hline Bronchoscopy & .0023 & & \\
\hline $\begin{array}{l}\text { Absence of purulent } \\
\text { secretions }\end{array}$ & .95 & & \\
\hline $\begin{array}{l}\text { Absence of repooling } \\
\text { secretions }\end{array}$ & .83 & & \\
\hline Best $\mathrm{PaO}_{2}$ & .0055 & & \\
\hline \multicolumn{4}{|l|}{ Chest CT imaging findings } \\
\hline Emphysema & .010 & .023 & $0.09(0.01-0.72)$ \\
\hline Traumatic Lung Injury & .027 & & \\
\hline Pneumothorax & .49 & & \\
\hline Pneumonia & .77 & & \\
\hline Aspiration & .82 & & \\
\hline Pleural Effusion & .46 & & \\
\hline Mucus Plugging & .33 & & \\
\hline Atelectasis & .30 & & \\
\hline
\end{tabular}

Analysis includes potential donors presenting to MTS with an $\mathrm{iPaO}_{2}>300 \mathrm{~mm} \mathrm{Hg}$. Bold font denotes $P$ values less than .05 . OR, Odds ratio; $C I$, confidence interval; $B M I$, body mass index; $C T$, computed tomography; $\mathrm{PaO}_{2}$, partial pressure of oxygen; MTS, Mid-America Transplant. *Includes potential donors with $\geq 20$ pack-year smoking history.
TABLE E3. Univariate and multivariate logistic regression analyses of likelihood of lung donation in potential donors with suboptimal $\mathrm{iPaO}_{2}$

\begin{tabular}{|c|c|c|c|}
\hline \multirow[b]{2}{*}{ Variable } & \multirow{2}{*}{$\frac{\text { Univariate }}{P \text { value }}$} & \multicolumn{2}{|c|}{ Multivariate } \\
\hline & & $P$ value & OR $(95 \%$ CI $)$ \\
\hline \multicolumn{4}{|l|}{ Demographic } \\
\hline Age, y & $<.0001$ & & \\
\hline Female sex & .88 & & \\
\hline BMI & $<.0001$ & & \\
\hline Asthma & .23 & & \\
\hline Smoking history* & $<.0001$ & .0001 & $0.33(0.18-0.58)$ \\
\hline Chest CT imaging & .60 & & \\
\hline \multicolumn{4}{|l|}{ In-patient history } \\
\hline Current antibiotic therapy & .0055 & & \\
\hline Sputum grain stain positive & .21 & & \\
\hline Sputum culture positive & .43 & & \\
\hline Bronchoscopy & $<.0001$ & & \\
\hline $\begin{array}{l}\text { Absence of purulent } \\
\text { secretions }\end{array}$ & .83 & & \\
\hline $\begin{array}{l}\text { Absence of repooling } \\
\text { secretions }\end{array}$ & .0001 & & \\
\hline Best $\mathrm{PaO}_{2}$ & $<.0001$ & $<.0001$ & $1.02(1.01-1.02)$ \\
\hline \multicolumn{4}{|l|}{ Chest CT imaging findings } \\
\hline Emphysema & .0012 & & \\
\hline Traumatic lung injury & .34 & & \\
\hline Pneumothorax & .46 & & \\
\hline Pneumonia & .77 & & \\
\hline Aspiration & .32 & & \\
\hline Pleural effusion & .20 & & \\
\hline Mucus plugging & .49 & & \\
\hline Atelectasis & .82 & & \\
\hline
\end{tabular}

Analysis includes potential donors presenting to MTS with an initial $\mathrm{PaO}_{2} \leq 300 \mathrm{~mm}$ $\mathrm{Hg}$. Bold font denotes $P$ values less than .05. OR, Odds ratio; $C I$, confidence interval $B M I$, body mass index; $C T$, computed tomography; $\mathrm{PaO}_{2}$, partial pressure of oxygen; MTS, Mid-America Transplant. *Includes potential donors with $\geq 20$ pack-year smoking history. 